\title{
Lowering and raising operators for the free Meixner class of orthogonal polynomials
}

Eugene Lytvynov

Department of Mathematics, Swansea University, Singleton Park, Swansea SA2 8PP, U.K.

e-mail: e.lytvynov@swansea.ac.uk

Irina Rodionova

Department of Mathematics, Swansea University, Singleton Park, Swansea SA2 8PP, U.K.

e-mail: i.rodionova@swansea.ac.uk

\begin{abstract}
We compare some properties of the lowering and raising operators for the classical and free classes of Meixner polynomials on the real line.

2000 AMS Mathematics Subject Classification: 42C05, 47B36

Keywords: Free Meixner class of orthogonal polynomials; Meixner class of orthogonal polynomials
\end{abstract}

\section{Classical and free Meixner classes}

In this note, we will compare some properties of the lowering and raising operators for the classical and free classes of Meixner polynomials on the real line.

Recall that the classical Meixner class consists of all monic orthogonal polynomials $\left(P_{n}(x)\right)_{n=0}^{\infty}$ whose exponential generating function has the form

$$
\sum_{n=0}^{\infty} \frac{z^{n}}{n !} P_{n}(x)=\exp (x \Psi(z)) f(z)=: G(x, z),
$$

where $z$ is from a neighborhood of zero in $\mathbb{C}, f$ and $\Psi$ are analytic functions in a neighborhood of zero such that $f(0)=1, \Psi(0)=\Psi^{\prime}(0)=0$, and the measure of orthogonality of $\left(P_{n}(x)\right)_{n=0}^{\infty}$, denoted by $\mu$, has infinite support [17] (see also [13]).

Recall that, given a finite set $A$, a set partition of $A$ is a collection $\left\{A_{1}, \ldots, A_{m}\right\}$ $(m \in \mathbb{N})$ of non-empty, mutually disjoint subsets of $A$ whose union is $A$. We denote by $\mathcal{P}_{n}$ the collection of all set partitions of the set $\{1,2, \ldots, n\}$. For any random variables $X_{1}, \ldots, X_{n}$ on a probability space $(\Omega, \mathcal{A}, P)$ which have all their moments finite, the cumulant of $X_{1}, \ldots, X_{n}$, denoted by $C_{n}\left(X_{1}, \ldots, X_{n}\right)$, is defined recurrently through the formula

$$
\mathbb{E}\left(X_{1} \cdots X_{n}\right)=\sum_{\pi \in \mathcal{P}_{n}} \prod_{A \in \pi} C\left(A, X_{1}, \ldots, X_{n}\right),
$$


where for any $A=\left\{i_{1}, \ldots, i_{k}\right\} \subset\{1, \ldots, n\}$

$$
C\left(A, X_{1}, \ldots, X_{n}\right):=C_{k}\left(X_{i_{1}}, \ldots, X_{i_{k}}\right) .
$$

Then the cumulant generating function of a random variable $X$ is defined by

$$
C_{X}(z)=\sum_{n=1}^{\infty} \frac{z^{n}}{n !} C_{n}(X, \ldots, X),
$$

where $z$ is from a neighborhood of zero in $\mathbb{C}$ for which the series in (44) converges absolutely. For a probability measure $\rho$ on $(\mathbb{R}, \mathcal{B}(\mathbb{R}))$ whose Laplace transform is analytic in a neighborhood of zero, the cumulant transform of $\rho$, denoted by $C_{\rho}(z)$, is defined as $C_{X}(z)$, where the random variable $X$ has distribution $\rho$. In fact, we have:

$$
C_{\rho}(z)=\log \left(\int_{\mathbb{R}} e^{z x} \rho(d x)\right) .
$$

The above assumptions on the polynomials from the Meixner class automatically imply that the generating function $G(x, z)$ can be represented as

$$
G(x, z)=\exp \left(x \Psi(z)-C_{\mu}(\Psi(z))\right)
$$

As shown in [17], a system of orthogonal polynomials $\left(P_{n}(x)\right)_{n=0}^{\infty}$ belongs to the Meixner class if and only if there exist $l \in \mathbb{R}, \lambda \in \mathbb{R}, t>0$, and $\eta \geq 0$ such that the $\left(P_{n}(x)\right)_{n=0}^{\infty}$ satisfy the recurrence relation

$$
x P_{n}(x)=P_{n+1}(x)+(\lambda n-l) P_{n}(x)+n(t+\eta(n-1)) P_{n-1}(x), \quad n \in \mathbb{N}_{0}:=\mathbb{N} \cup\{0\},
$$

where $P_{0}(x)=1$ and $P_{-1}(x)=0$. It is easy to see that, if we set $l=0$ in (6) , the corresponding measure $\mu$ will become centered, whereas $l \neq 0$ corresponds to the shift of the centered measure by $l$. Therefore, we will restrict our attention to centered measures, by setting $l=0$.

One has to distinguish the five following cases:

a) $\lambda=\eta=0, \mu$ is Gaussian measure and $\left(P_{n}(x)\right)_{n=0}^{\infty}$ are Hermite polynomials;

b) $\lambda \neq 0$ and $\eta=0, \mu$ is centered Poisson measure, $\left(P_{n}(x)\right)_{n=0}^{\infty}$ are Charlier polynomials;

c) $\eta>0$ and $\lambda^{2}=4 \eta, \mu$ is centered gamma measure, $\left(P_{n}(x)\right)_{n=0}^{\infty}$ are Laguerre polynomials;

d) $\eta>0$ and $\lambda^{2}>4 \eta, \mu$ is centered Pascal measure, $\left(P_{n}(x)\right)_{n=0}^{\infty}$ are Meixner polynomials of the first kind;

e) $\eta>0$ and $\lambda^{2}<4 \eta, \mu$ is Meixner measure, $\left(P_{n}(x)\right)_{n=0}^{\infty}$ are Meixner polynomials of the second kind, or Meixner-Polaczek polynomials in other terms. 
In what follows, we will denote by $\mu_{\lambda, \eta}^{(t)}, G_{\lambda, \eta}^{(t)}, \Psi_{\lambda, \eta}^{(t)}$, and $C_{\lambda, \eta}^{(t)}$ the measure $\mu$ and the functions $G, \Psi$, and $C_{\mu}$, respectively, which correspond to the choice of the parameters $\lambda, \eta$, and $t$ as in (6). For $t=1$, we will usually skip the upper index ${ }^{(1)}$.

For any possible choice of $\lambda$ and $\eta$, and for any $t>0$, we have $\Psi_{\lambda, \eta}^{(t)}(z)=\Psi_{\lambda, \eta}(z)$, $C_{\lambda, \eta}^{(t)}(z)=t C_{\lambda, \eta}(z)$, so that

$$
G_{\lambda, \eta}^{(t)}(x, z)=\exp \left(x \Psi_{\lambda, \eta}(z)-t C_{\lambda, \eta}\left(\Psi_{\lambda, \eta}(z)\right)\right) .
$$

Furthermore, for any $\lambda$ and $\eta$ as above, there exists a Lévy process $X_{\lambda, \eta}=\left(X_{\lambda, \eta}(t)\right)_{t \geq 0}$ such that $X_{\lambda, \eta}(0)=0$ a.s., and for each $t>0$ the random variable $X_{\lambda, \eta}(t)$ has distribution $\mu_{\lambda, \eta}^{(t)}$ (see e.g. [20]).

Let $\nu_{\lambda, \eta}$ be the probability measure on $\mathbb{R}$ which is the measure of orthogonality of the polynomials $\left(Q_{n}(x)\right)_{n=0}^{\infty}$ satisfying

$$
x Q_{n}(x)=Q_{n+1}(x)+\lambda(n+1) Q_{n}(x)+\eta n(n+1) Q_{n-1}(x), \quad n \in \mathbb{N}_{0},
$$

with $Q_{0}(x)=1, Q_{-1}(x)=0$.

Thus, if $\eta=0$, then $\nu_{\lambda, 0}=\delta_{\lambda}$ (the Dirac measure with mass at $\lambda$ ), while for $\eta>0$ $\nu_{\lambda, \eta}$ is the measure of orthogonality of polynomials from the Meixner class satisfying (6) with $\lambda, \eta$ as above, $l=-\lambda$ and $t=2 \eta$. Then, for $z$ from a neighborhood of zero in $\mathbb{C}$, we have:

$$
\begin{aligned}
C_{\lambda, \eta}(z) & =\int_{\mathbb{R}}\left(e^{s z}-1-s z\right) s^{-2} \nu_{\lambda, \eta}(d s) \\
& =\int_{\mathbb{R}} \sum_{n=2}^{\infty} \frac{s^{n-2} z^{n}}{n !} \nu_{\lambda, \eta}(d s),
\end{aligned}
$$

i.e., $s^{-2} \nu_{\lambda, \eta}(d s)$ is the Lévy measure of the Lévy process $X_{\lambda, \eta}$.

In free probability, Meixner's class of orthogonal polynomials was introduced and studied by Anshelevich [2] (see also [4]) and Saitoh, Yoshida [19]. (In fact, such polynomials had already occurred in many places in the literature even before [2, 19], see [9, p. 62] and [4, p. 864] for bibliographical references.) A deep study of multivariate free Meixner polynomials of non-commutative variables has been carried out by Anshelevich, see [3, 4, 5] and the references therein. Bożejko and Bryc [9] gave a characterization of free Meixner polynomials in terms of a regression problem. We also refer to [2, 3, 10] for $q$-interpolation of the classical and free Meixner classes.

Below, in the free case, for many objects we will use the same notations as those used for their counterparts in the classical case. However, it should always be clear from the context which objects are being meant.

The free Meixner class consists of all monic orthogonal polynomials $\left(P_{n}(x)\right)_{n=0}^{\infty}$ whose (usual) generating function has the form

$$
\sum_{n=0}^{\infty} z^{n} P_{n}(x)=(1-x \Psi(z))^{-1} f(z)=: G(x, z),
$$


where $z, \Psi$ and $f$ satisfy the same assumptions as in the classical case, and the measure of orthogonality $\mu$ has infinite support. Recall the following notation from $q$-analysis: for each $q \in[-1,1]$, we define $[0]_{q}:=0$ and $[n]_{q}:=1+q+q^{2}+\cdots+q^{n-1}$, for $n \in \mathbb{N}$, and $[n]_{q} !:=[1]_{q}[2]_{q} \cdots[n]_{q}$. In particular, the free probability corresponds to $q=0$, in which case $[0]_{0}=0$ and $[n]_{0}=1$ for $n \in \mathbb{N}$. Thus the free analog of $\exp (x)=\sum_{n=0}^{\infty} \frac{x^{n}}{n !}$ is the resolvent function $(1-x)^{-1}=\sum_{n=0}^{\infty} x^{n}$, so that (10) is indeed the free analog of (1).

Consider a non-commutative probability space which is a von Neumann algebra $\mathcal{A}$ with a normal, faithful, tracial state $\tau: \mathcal{A} \rightarrow \mathbb{C}$, i.e., $\tau(\cdot)$ is linear, continuous in weak* topology, $\tau(a b)=\tau(b a), \tau(\mathrm{id})=1, \tau\left(a a^{*}\right) \geq 0$, and $\tau\left(a a^{*}\right)=0$ implies $a=0$. A (non-commutative) random variable $X$ is a self-adjoint element of $\mathcal{A}$.

We denote by $\mathcal{N C}(n)$ the collection of all non-crossing partitions of $\{1, \ldots, n\}$, i.e., all set partitions $\pi=\left\{A_{1}, \ldots, A_{k}\right\}, k \geq 1$, of $\{1, \ldots, n\}$ such that there do not exist $A_{i}, A_{j} \in \pi, A_{i} \neq A_{j}$, for which the following inequalities hold: $x_{1}<y_{1}<x_{2}<y_{2}$ for some $x_{1}, x_{2} \in A_{i}$ and $y_{1}, y_{2} \in A_{j}$. For any non-commutative random variables $X_{1}, \ldots, X_{n}$, the free cumulant $C_{n}\left(X_{1}, \ldots, X_{n}\right)$ is defined through formulas (2n), (3) in which $\mathbb{E}$ is replaced by $\tau$, and $\mathcal{P}_{n}$ is replaced by $\mathcal{N C}(n)$. Then, analogously to (44), the free cumulant generating function of a random variable $X$ is defined by

$$
C_{X}(z)=\sum_{n=1}^{\infty} z^{n} C_{n}(X, \ldots, X) .
$$

If $\rho$ is a probability measure on $(\mathbb{R}, \mathcal{B}(\mathbb{R}))$ with compact support, we define the free cumulant transform $C_{\rho}(z)$ of $\rho$ as $C_{X}(z)$, where a random variable $X$ has distribution $\rho$, i.e., $\tau\left(X^{n}\right)=\int_{\mathbb{R}} x^{n} \rho(d x)$.

By [2], the generating function of the fee Meixner polynomials can be represented as

$$
G(x, z)=\left(1-x \Psi(z)+C_{\mu}(\Psi(z))\right)^{-1}
$$

(compare with (5)). Furthermore, a system of orthogonal polynomials $\left(P_{n}(x)\right)_{n=0}^{\infty}$ belongs to the free Meixner class if and only if there exist $l \in \mathbb{R}, \lambda \in \mathbb{R}, t>0$, and $\eta \geq 0$ such that the $\left(P_{n}(x)\right)_{n=0}^{\infty}$ satisfy the recurrence relation

$$
x P_{n}(x)=P_{n+1}(x)+\left(\lambda[n]_{0}-l\right) P_{n}(x)+[n]_{0}\left(t+\eta[n-1]_{0}\right) P_{n-1}(x),
$$

and we again set $l=0$ in order to set the measure $\mu$ centered. Analogously to the classical case, we thus again have five classes of free Meixner polynomials. Next, analogously to (7), we have

$$
G_{\lambda, \eta}^{(t)}(x, z)=\left(1-x \Psi_{\lambda, \eta}(z)+t C_{\lambda, \eta}\left(\Psi_{\lambda, \eta}(z)\right)\right)^{-1}
$$

(we have used obvious notations). 
Recall that non-commutative random variables $X_{1}, \ldots, X_{n}$ are called freely independent if, for any $k \geq 1$ and any non-constant choice of $Y_{1}, \ldots, Y_{k} \in\left\{X_{1}, \ldots, X_{n}\right\}$, $C_{k}\left(Y_{1}, \ldots, Y_{k}\right)=0$ [22] (see also [9]). A non-commutative stochastic process $X=$ $(X(t))_{t \geq 0}$ is called a free Lévy process if $X_{0}=0$ and the increments of $X$ are freely independent and stationary, see [8] for details.

By [2, 19, for any allowed parameters $\lambda$ and $\eta$, there exists a free Lévy process $X_{\lambda, \eta}=\left(X_{\lambda, \eta}(t)\right)_{t \geq 0}$ such that, for each $t>0$, the random variable $X_{\lambda, \eta}(t)$ has distribution $\mu_{\lambda, \eta}^{(t)}$. Let $\nu_{\lambda, \eta}$ be the probability measure on $\mathbb{R}$ which is the measure of orthogonality of the polynomials $\left(Q_{n}(x)\right)_{n=0}^{\infty}$ satisfying

$$
\begin{aligned}
x Q_{n}(x) & =Q_{n+1}(x)+\lambda[n+1]_{0} Q_{n}(x)+\eta[n]_{0}[n+1]_{0} Q_{n-1}(x), \\
& =Q_{n+1}(x)+\lambda Q_{n}(x)+\eta Q_{n-1}(x),
\end{aligned}
$$

with $Q_{0}(x)=1, Q_{-1}(x)=0$. Thus, if $\eta=0$, then $\nu_{\lambda, 0}=\delta_{\lambda}$, while for $\eta>0$

$$
\nu_{\lambda, \eta}=\mu_{\lambda, 0}^{(\eta)} .
$$

(Thus, for $\eta>0, \nu_{\lambda, \eta}$ is the free Gaussian distribution if $\lambda=0$ and the free Poisson distribution if $\lambda \neq 0$.) Then, for $z$ from a neighborhood of zero in $\mathbb{C}$, we have:

$$
\begin{aligned}
C_{\lambda, \eta}(z) & =\int_{\mathbb{R}}\left((1-s z)^{-1}-1-s z\right) s^{-2} \nu_{\lambda, \eta}(d s) \\
& =\int_{\mathbb{R}} \sum_{n=2}^{\infty} z^{n} s^{n-2} \nu_{\lambda, \eta}(d s),
\end{aligned}
$$

i.e., $s^{-2} \nu_{\lambda, \eta}(d s)$ is the free Lévy measure of the free Lévy process $X_{\lambda, \eta}[6$.

Remark 1.1. The polynomials of the classical Mexiner class with $\eta>0$ naturally appear in the study of a realization of the renormalized square of white noise, see [1]. In fact, as it (indirectly) follows from [21] (see also [11), the polynomials of the free Meixner class with $\eta>0$ are related to the renormalized square of free white noise.

\section{Raising and lowering operators}

We fix any $\lambda \in \mathbb{R}$ and $\eta \geq 0$. In the case of classical Meixner polynomials, we define the raising and lowering operators, $\partial_{\lambda, \eta}^{\dagger}$ and $\partial_{\lambda, \eta}$, as linear operators given through

$$
\left(\partial_{\lambda, \eta}^{\dagger} P_{n}\right)(x)=P_{n+1}(x), \quad\left(\partial_{\lambda, \eta} P_{n}\right)(x)=n P_{n-1}(x),
$$

where $\left(P_{n}(x)\right)_{n=0}^{\infty}$ satisfy (6) (with $t=1$ and $l=0$ ). We will denote by $x$ the operator of multiplication by variable $x$ in $L^{2}\left(\mathbb{R}, \mu_{\lambda, \eta}\right)$. By (6) , we then have

$$
x=\partial_{\lambda, \eta}^{\dagger}\left(1+\lambda \partial_{\lambda, \eta}+\eta \partial_{\lambda, \eta}^{2}\right)+\partial_{\lambda, \eta} .
$$


Since the operators $\partial_{\lambda, \eta}^{\dagger}, \partial_{\lambda, \eta}$ are unbounded in $L^{2}\left(\mathbb{R}, \mu_{\lambda, \eta}\right)$, we will later identify their domain.

In the free case, we define the raising and lowering operators, $\partial_{\lambda, \eta}^{\dagger}$ and $\partial_{\lambda, \eta}$ through

$$
\left(\partial_{\lambda, \eta}^{\dagger} P_{n}\right)(x)=P_{n+1}(x), \quad\left(\partial_{\lambda, \eta} P_{n}\right)(x)=[n]_{0} P_{n-1}(x),
$$

where $\left(P_{n}(x)\right)_{n=0}^{\infty}$ satisfy (12) (again with $t=1$ and $l=0$ ). The operator of multiplication by $x$ then has the same representation (17) in $L^{2}\left(\mathbb{R}, \mu_{\lambda, \eta}\right)$. Note that the operators $x, \partial_{\lambda, \eta}^{\dagger}$, and $\partial_{\lambda, \eta}$ are now bounded.

\section{$2.1 \quad$ Lowering operator}

We start with the classical case. So, denote by $\mathcal{P}$ the set of all polynomials on $\mathbb{R}$. For each $f(x)=\sum_{n=0}^{N} f_{n} P_{n}(x) \in \mathcal{P}, n \in \mathbb{N}_{0}$, and each $q \in \mathbb{N}$, we set

$$
\|f\|_{q}^{2}=\sum_{n=0}^{\infty}\left|f_{n}\right|^{2}(n !)^{2} 2^{n q} .
$$

Let $H_{q}$ denote the Hilbert space obtained as the completion of $\mathcal{P}$ in the $\|\cdot\|_{q}$ norm. We then define the nuclear space $\Phi:=\operatorname{proj} \lim _{q \rightarrow \infty} H_{q}$. Since $\left(P_{n}(x)\right)_{n=0}^{\infty}$ is a Scheffer system of polynomials, there exists $q \in \mathbb{N}$ such that $H_{q}$ (and so $\Phi$ ) is continuously embedded into $L^{2}\left(\mathbb{R}, \mu_{\lambda, \eta}\right)$ (see [14]).

Denote by $\mathcal{E}_{\min }^{1}(\mathbb{C})$ the set of all entire functions on $\mathbb{C}$ of first order of growth and of minimal type, i.e., a function $f$ entire on $\mathbb{C}$ belongs to $\mathcal{E}_{\text {min }}^{1}(\mathbb{C})$ if and only if for each $\varepsilon>0$ there exists $C>0$ such that $|f(z)| \leq C \exp (\varepsilon|z|)$. Denote by $\mathcal{E}_{\text {min }}^{1}(\mathbb{R})$ the set of restrictions to $\mathbb{R}$ of functions from $\mathcal{E}_{\text {min }}^{1}(\mathbb{C})$. Following [14, 15], we then introduce a norm on $\mathcal{E}_{\text {min }}^{1}(\mathbb{C})$, and hence on $\mathcal{E}_{\text {min }}^{1}(\mathbb{R})$, as follows. Each $f \in \mathcal{E}_{\text {min }}^{1}(\mathbb{C})$ can be uniquely represented in the form $f(z)=\sum_{n=0}^{\infty} f_{n} z^{n}$, and we set, for any $q \in \mathbb{N}$,

$$
N_{q}(f):=\sum_{n=0}^{\infty}\left|f_{n}\right|^{2}(n !)^{2} 2^{n q} .
$$

By [14, Theorems 2.5, 3.8 and subsec. 6.2], $\Phi=\mathcal{E}_{\min }^{1}(\mathbb{R})$ and the two systems of norms on $\Phi:\left(\|\cdot\|_{q}, q \in \mathbb{N}\right)$ and $\left(N_{q}(\cdot), q \in \mathbb{N}\right)$ are equivalent, and hence determine the same topology on $\Phi$.

Using the $\|\cdot\|_{q}$ norms, we easily conclude that the operators $\partial_{\lambda, \eta}^{\dagger}$ and $\partial_{\lambda, \eta}$ act continuously on $\mathcal{E}_{\min }^{1}(\mathbb{R})$. We have the following theorem which describes the action of $\partial_{\lambda, \eta}$ in the classical case (compare with [18, Theorem 2.2] and [16, Theorem 4.1]):

Theorem 2.1. In the classical case, we have, for any $f \in \mathcal{E}_{\min }^{1}(\mathbb{R})$ :

$$
\left(\partial_{\lambda, \eta} f\right)(x)=\int_{\mathbb{R}} \frac{f(x+s)-f(x)}{s} \nu_{\lambda, \eta}(d s), \quad x \in \mathbb{R} .
$$


Remark 2.1. In the Gaussian case, i.e., when $\lambda=\eta=0$, we have $\nu_{0,0}=\delta_{0}$ and formula (18) is understood in the limiting sense: $\left(\partial_{0,0} f\right)(x)=(D f)(x):=f^{\prime}(x)$.

Remark 2.2. In the Poisson case, i.e., when $\eta=0$ and $\lambda \neq 0$, we have $\nu_{\lambda, 0}=\delta_{\lambda}$, so that

$$
\left(\partial_{\lambda, 0} f\right)(x)=\frac{f(x+\lambda)-f(x)}{\lambda} .
$$

Therefore, if $\eta \neq 0$,

$$
\left(\partial_{\lambda, \eta} f\right)(x)=\int_{\mathbb{R}}\left(\partial_{\lambda, 0} f\right)(x) \nu_{\lambda, \eta}(d \lambda)
$$

and so the operator $\partial_{\lambda, \eta}$ is the $\nu_{\lambda, \eta}$-mixture of the operators $\partial_{\lambda, 0}$.

Proof of Theorem 2.1. For each $q \in \mathbb{N}$, denote by $G_{q}$ the Hilbert space obtained as the completion of $\mathcal{E}_{\text {min }}^{1}(\mathbb{R})$ in the $N_{q}(\cdot)$ norm. As easily seen, the operator $\partial_{\lambda, \eta}$ acts continuously in each $H_{q}$. Hence, there exist $q_{1} \geq q_{2} \geq 1$ such that $G_{q_{2}}$ is continuously embedded into $L^{2}\left(\mathbb{R}, \mu_{\lambda, \eta}\right)$ and $\partial_{\lambda, \eta}: G_{q_{1}} \rightarrow G_{q_{2}}$ is a continuous operator. Choose $\varepsilon>0$ such that, for each $z \in \mathbb{R},|z|<\varepsilon, e^{x z}$ and $e^{x \Psi_{\lambda, \eta}(z)}$ belong to $G_{q_{1}}$ as functions of $x$. Hence, $G(\cdot, z) \in G_{q_{1}}$ and

$$
\left(\partial_{\lambda, \eta} G_{\lambda, \eta}(\cdot, z)\right)(x)=z G_{\lambda, \eta}(x, z)
$$

which implies

$$
\partial_{\lambda, \eta} e^{x z}=\Psi_{\lambda, \eta}^{-1}(z) e^{x z}
$$

On the other hand, by (9),

$$
\begin{aligned}
\int_{\mathbb{R}} \frac{e^{(x+s) z}-e^{x z}}{s} \nu_{\lambda, \eta}(d s) & =e^{x z} \int_{\mathbb{R}} \frac{e^{s z}-1}{s} \nu_{\lambda, \eta}(d s) \\
& =e^{x z} C_{\lambda, \eta}^{\prime}(z) .
\end{aligned}
$$

By [5, Proposition 1] (see also [17]), we have

$$
\Psi_{\lambda, \eta}^{-1}(z)=C_{\lambda, \eta}^{\prime}(z)
$$

Hence, (19)-(21) imply that (18) holds when $f(x)=e^{x z},|z|<\varepsilon$.

Since $\int_{\mathbb{R}} e^{\varepsilon|s|} \nu_{\lambda, \eta}(d s)<\infty$, there exits $C \geq 1$ such that, for all $n \in \mathbb{N}$,

$$
\int_{\mathbb{R}}|s|^{n} \nu_{\lambda, \eta}(d s) \leq C^{n} n !
$$

Hence, for each $f(x)=\sum_{n=0}^{\infty} f_{n} x^{n} \in \mathcal{E}_{\text {min }}^{1}(\mathbb{R})$ and $x \in \mathbb{R}$,

$$
\int_{\mathbb{R}}\left|\frac{f(x+s)-f(x)}{s}\right| \nu_{\lambda, \eta}(d s) \leq \sum_{n=1}^{\infty}\left|f_{n}\right| \int_{\mathbb{R}}\left|\frac{(x+s)^{n}-x^{n}}{s}\right| \nu_{\lambda, \eta}(d s)
$$




$$
\begin{aligned}
& \leq \sum_{n=1}^{\infty}\left|f_{n}\right| \sum_{k=0}^{n-1}\left(\begin{array}{l}
n \\
k
\end{array}\right)|x|^{k} \int_{\mathbb{R}}|s|^{n-k-1} \nu_{\lambda, \eta}(d s) \\
& \leq \sum_{n=1}^{\infty}\left|f_{n}\right| n ! n C^{n}(1 \vee|x|)^{n} \\
& \leq N_{q_{3}}(f)\left(\sum_{n=1}^{\infty} 4^{-n}\right)^{1 / 2}<\infty,
\end{aligned}
$$

where $q_{3}=q_{3}(x) \in \mathbb{N}$ is chosen so that $q_{3} \geq q_{2}$ and $(4 C(1 \vee|x|))^{2} \leq 2^{q_{3}}$.

Now, let $\left\{f^{(k)}\right\}_{k=1}^{\infty} \subset G_{q_{3}}$ be such that each $f^{(k)}$ is a linear combination of functions $e^{x z}$ with $|z|<\varepsilon$ and $f^{(k)} \rightarrow f$ as $k \rightarrow \infty$ in $G_{q_{3}}$. Then, analogously to (22), we conclude that

$$
\int_{\mathbb{R}} \frac{f^{(k)}(x+s)-f^{(k)}(x)}{s} \nu_{\lambda, \eta}(d s) \rightarrow \int_{\mathbb{R}} \frac{f(x+s)-f(x)}{s} \nu_{\lambda, \eta}(d s),
$$

and since $\partial_{\lambda, \eta} f^{(k)} \rightarrow \partial_{\lambda, \eta} f$ in $G_{q_{1}},\left(\partial_{\lambda, \eta} f^{(k)}\right)(x) \rightarrow\left(\partial_{\lambda, \eta} f\right)(x)$. From here the theorem follows.

We will now derive a free counterpart of Theorem 2.1. By the theory of Jacobi matrices (see e.g. [7]), in the free case, the measure $\mu_{\lambda, \eta}^{(t)}$ is concentrated on the interval $[-(\lambda \vee \sqrt{\eta+t}),(\lambda \vee \sqrt{\eta+t})]$. Below $C^{1}([a, b])$ denotes the set of all continuously differentiable functions on interval $[a, b]$.

Theorem 2.2. In the free case, we have for any $f \in C^{1}\left(\left[-r_{\lambda, \eta}, r_{\lambda, \eta}\right]\right)$,

$$
\begin{aligned}
\left(\partial_{\lambda, \eta} f\right)(x) & =\int_{\left[-r_{\lambda, \eta}, r_{\lambda, \eta}\right]} \frac{f(x)-f(s)}{x-s} \nu_{\lambda, \eta+1}(d s) \\
& =\int_{\left[-r_{\lambda, \eta}, r_{\lambda, \eta}\right]} \frac{f(x)-f(s)}{x-s} \mu_{\lambda, 0}^{(\eta+1)}(d s), \quad x \in \mathbb{R} .
\end{aligned}
$$

Here, $r_{\lambda, \eta}:=(\lambda \vee \sqrt{\eta+1})$.

Remark 2.3. Unlike in the classical case, the integral representation of the operator $\partial_{\lambda, \eta}$ in the free case uses the measure $\nu_{\lambda, \eta+1}$ with the 'shifted' parameter $\eta+1$. So, in particular, in the free Gaussian case $(\lambda=\eta=0), \partial_{0,0}$ is not the operator of free differentiation $f(x) \mapsto\left(D_{\text {free }} f\right)(x)=\frac{f(x)-f(0)}{x}$. In fact, in the free Gaussian and free Poisson cases, i.e., when $\eta=0$, the integration on the right hand side of formula (24) is with respect to the measure of orthogonality $\mu_{\lambda, 0}$. Thus, in particular,

$$
P_{n-1}(x)=\int_{\left[-r_{\lambda, 0}, r_{\lambda, 0}\right]} \frac{P_{n}(x)-P_{n}(s)}{x-s} \mu_{\lambda, 0}(d s),
$$

where $\left(P_{n}(x)\right)_{n=0}^{\infty}$ are orthogonal with respect to $\mu_{\lambda, 0}$. 
Remark 2.4. In the classical and free cases, denote by $m_{\lambda, \eta}(n)$ the $n$-th moment of $\nu_{\lambda, \eta}$ :

$$
m_{\lambda, \eta}(n):=\int_{\mathbb{R}} s^{n} \nu_{\lambda, \eta}(d s) .
$$

Then, by Theorem 2.1,

$$
\partial_{\lambda, \eta} x^{n}=\sum_{k=0}^{n-1}\left(\begin{array}{l}
n \\
k
\end{array}\right) m_{\lambda, \eta}(n-1-k) x^{k},
$$

while in the fee case, by Theorem 2.2 ,

$$
\partial_{\lambda, \eta} x^{n}=\sum_{k=0}^{n-1} m_{\lambda, \eta+1}(n-1-k) x^{k}
$$

Recall the the free $(q=0)$ analog of the binomial coefficient $\left(\begin{array}{l}n \\ k\end{array}\right)$ is 1 .

Proof of Theorem 2.2. First, we note that, by (15), formulas (23) and (24) are equivalent. By [2], for sufficiently small $z$

$$
\begin{aligned}
\Psi_{\lambda, \eta}(z) & =\frac{z}{1+\lambda z+\eta z^{2}}, \\
C_{\lambda, \eta}\left(\Psi_{\lambda, \eta}(z)\right) & =\frac{z^{2}}{1+\lambda z+\eta z^{2}} .
\end{aligned}
$$

Hence, by (11), we easily see that

$$
\begin{aligned}
G_{\lambda, \eta}(x, z) & =\left(1-\frac{x z-z^{2}}{1+\lambda z+\eta z^{2}}\right)^{-1} \\
& =\frac{1+\lambda z+\eta z^{2}}{1+\lambda z+(\eta+1) z^{2}}\left(1-x \Psi_{\lambda, \eta+1}(z)\right)^{-1}
\end{aligned}
$$

Since

$$
\left(\partial_{\lambda, \eta} G_{\lambda, \eta}(\cdot, z)\right)(x)=z G_{\lambda, \eta}(x, z)
$$

we, therefore, have

$$
\partial_{\lambda, \eta}(1-x z)^{-1}=\Psi_{\lambda, \eta+1}^{-1}(z)(1-x z)^{-1} .
$$

Next, by (16)

$$
\begin{aligned}
& \int_{\left[-r_{\lambda, \eta}, r_{\lambda, \eta}\right]} \frac{(1-x z)^{-1}-(1-s z)^{-1}}{x-s} \nu_{\lambda, \eta+1}(d s) \\
& =(1-x z)^{-1} \int_{\left[-r_{\lambda, \eta}, r_{\lambda, \eta}\right]} z(1-s z)^{-1} \nu_{\lambda, \eta+1}(d s)
\end{aligned}
$$




$$
\begin{aligned}
& =(1-x z)^{-1} z^{-1} \int_{\left[-r_{\lambda, \eta}, r_{\lambda, \eta}\right]} \sum_{n=2}^{\infty} z^{n} s^{n-2} \nu_{\lambda, \eta+1}(d s) \\
& =(1-x z)^{-1} z^{-1} C_{\lambda, \eta+1}(z) .
\end{aligned}
$$

By [5, Proposition 1] (see also [2]),

$$
\Psi_{\lambda, \eta}^{-1}(z)=z^{-1} C_{\lambda, \eta}(z)
$$

(compare with (21) and note that $z^{-1} C_{\lambda, \eta}(z)$ is the free derivative of $C_{\lambda, \eta}$ ). By (27)(29), equality (23) holds for $f(x)=(1-x z)^{-1}$ for all sufficiently small $z$. From here, the general case follows by an easy approximation argument.

Remark 2.5. Note that, in view of (19) and (27), in the classical case

$$
\partial_{\lambda, \eta}=\Psi_{\lambda, \eta}^{-1}(D)
$$

while in the free case

$$
\partial_{\lambda, \eta}=\Psi_{\lambda, \eta+1}^{-1}\left(D_{\text {free }}\right)
$$

\subsection{Raising operator}

We again start with the classical case (cf. [16, 18]). Following [17], we define $\alpha, \beta \in \mathbb{C}$ through the equation

$$
1+\lambda x+\eta x^{2}=(1-\alpha x)(1-\beta x)
$$

or equivalently

$$
\alpha+\beta=-\lambda, \quad \alpha \beta=\eta,
$$

where in the case $\eta=0$ and $\lambda \neq 0$ one sets $\alpha=-\lambda$ and $\beta=0$. Evidently, the condition $\lambda \in \mathbb{R}, \eta \geq 0$ is satisfied if and only if either $\alpha, \beta \in \mathbb{R}$ and $\alpha$ and $\beta$ are of the same sign, or $\operatorname{Im}(\alpha) \neq 0$ and $\alpha$ and $\beta$ are complex conjugate.

Theorem 2.3. In the classical case, there exists $\varepsilon>0$ such that, for all $z \in \mathbb{R},|z|<\varepsilon$, we have

$$
\begin{aligned}
\partial_{\lambda, \eta}^{\dagger} e^{x z}= & \left(\frac{x}{1+\lambda \Psi_{\lambda, \eta}^{-1}(z)+\eta\left(\Psi_{\lambda, \eta}^{-1}(z)\right)^{2}}-\frac{\Psi_{\lambda, \eta}^{-1}(z)}{1+\lambda \Psi_{\lambda, \eta}^{-1}(z)+\eta\left(\Psi_{\lambda, \eta}^{-1}(z)\right)^{2}}\right) e^{x z} \\
= & {\left[x e^{-z(\alpha-\beta)}\left(1+\frac{\alpha}{\alpha-\beta}\left(e^{z(\alpha-\beta)}-1\right)\right)^{2}\right.} \\
& \left.-e^{-z(\alpha-\beta)}\left(1+\frac{\alpha}{\alpha-\beta}\left(e^{z(\alpha-\beta)}-1\right)\right) \frac{1}{\alpha-\beta}\left(e^{z(\alpha-\beta)}-1\right)\right] e^{x z}
\end{aligned}
$$

for $\alpha=\beta$ the formula being understood in the limiting sense. 
Remark 2.6. Theorem 2.3 shows that the operator $\partial_{\lambda, \eta}^{\dagger}$ is represented through the operator of multiplication by $x$ and through analytic functions (in a neighborhood of zero) of the operator of differentiation $D$.

Proof of Theorem 2.3. By (17) and (19), we have

$$
x e^{x z}=\left(1+\lambda \Psi_{\lambda, \eta}^{-1}(z)+\eta\left(\Psi_{\lambda, \eta}^{-1}(z)\right)^{2}\right) \partial_{\lambda, \eta}^{\dagger} e^{x z}+\Psi_{\lambda, \eta}^{-1}(z) e^{x z},
$$

from where (31) follows. To derive (32) from (31), use (30) and the following formula (see [17]):

$$
\Psi_{\lambda, \eta}^{-1}(z)=\left(\frac{1}{\alpha-\beta}\left(e^{z(\alpha-\beta)}-1\right)\right)\left(1+\frac{\alpha}{\alpha-\beta}\left(e^{z(\alpha-\beta)}-1\right)\right)^{-1}
$$

Using formula (32) and analogously to the proof of Theorem 2.1, one easily derives explicit formulas for the action of $\partial_{\lambda, \eta}^{\dagger}$ (cf. [16, 18]). Before formulating this result, we introduce the following natations: For each $s \in \mathbb{R}, s \neq 0$ and for $f: \mathbb{R} \rightarrow \mathbb{R}$, we define

$$
\begin{aligned}
\left(\nabla_{s} f\right)(x) & :=\frac{f(x+s)-f(x)}{s}, \\
\left(U_{s} f\right)(x) & :=f(x+s) .
\end{aligned}
$$

Clearly, $D, \nabla_{s}$, and $U_{s}$ act continuously on $\mathcal{E}_{\text {min }}^{1}(\mathbb{R})$.

Corollary 2.1. We have the following representation of the operator $\partial_{\lambda, \eta}$ on $\mathcal{E}_{\min }^{1}(\mathbb{R})$ : for $\lambda=\eta=0$

$$
\partial_{0,0}^{\dagger}=x-D
$$

for $\lambda \neq 0$ and $\eta=0$

$$
\partial_{\lambda, 0}^{\dagger}=x\left(1-\lambda \nabla_{\lambda}\right)-\nabla_{\lambda}
$$

for $\eta>0$ and $\lambda^{2}=4 \eta$

$$
\partial_{\lambda, \eta}^{\dagger}=x(D-1)^{2}-D(D-1),
$$

and for $\eta>0$ and $\lambda^{2} \neq 4 \eta$

$$
\partial_{\lambda, \eta}^{\dagger}=x\left(1+\alpha \nabla_{\alpha-\beta}\right)^{2} U_{\beta-\alpha}-\left(1+\alpha \nabla_{\alpha-\beta}\right) \nabla_{\alpha-\beta} U_{\beta-\alpha} .
$$

We proceed to consider the free case.

Theorem 2.4. In the free case, there exists $\varepsilon>0$ such that, for all $z \in \mathbb{R},|z|<\varepsilon$, we have

$$
\begin{aligned}
& \partial_{\lambda, \eta}^{\dagger}(1-x z)^{-1} \\
& =\left(\frac{x}{1+\lambda \Psi_{\lambda, \eta+1}^{-1}(z)+\eta\left(\Psi_{\lambda, \eta+1}^{-1}(z)\right)^{2}}-\frac{\Psi_{\lambda, \eta+1}^{-1}(z)}{1+\lambda \Psi_{\lambda, \eta+1}^{-1}(z)+\eta\left(\Psi_{\lambda, \eta+1}^{-1}(z)\right)^{2}}\right)(1-x z)^{-1}
\end{aligned}
$$




$$
\begin{aligned}
=(x & \frac{4 z^{2}(\eta+1)^{2}}{\left(2 \eta+1+\lambda z+\sqrt{(1-\lambda z)^{2}-4 z^{2}(\eta+1)}\right)\left(1-\lambda z-\sqrt{(1-\lambda z)^{2}-4 z^{2}(\eta+1)}\right)} \\
& \left.+\frac{2 z(\eta+1)}{2 \eta+1+\lambda z+\sqrt{(1-\lambda z)^{2}-4 z^{2}(\eta+1)}}\right)(1-x z)^{-1} .
\end{aligned}
$$

Remark 2.7. By Theorem 2.4, the operator $\partial_{\lambda, \eta}^{\dagger}$ is represented through the operator of multiplication by $x$ and through analytic functions (in a neighborhood of zero) of the operator of free differentiation $D_{\text {free }}$.

Proof of Theorem 2.4. The derivation of (33) is analogous to the classical case. So, we only have to show that (34) holds. By (25) and (33),

$$
\partial_{\lambda, \eta}^{\dagger}(1-x z)^{-1}=\left(x \frac{\Psi_{\lambda, \eta}\left(\Psi_{\lambda, \eta+1}^{-1}(z)\right)}{\Psi_{\lambda, \eta+1}^{-1}(z)}-\Psi_{\lambda, \eta}\left(\Psi_{\lambda, \eta+1}^{-1}(z)\right)\right)(1-x z)^{-1}
$$

Next, by (25),

$$
\begin{aligned}
\frac{1}{\Psi_{\lambda, \eta+1}(z)} & =\frac{1+\lambda z+\eta z^{2}}{z}+z \\
& =\frac{1}{\Psi_{\lambda, \eta}(z)}+z .
\end{aligned}
$$

Hence,

$$
\frac{1}{z}=\frac{1}{\Psi_{\lambda, \eta+1}\left(\Psi_{\lambda, \eta+1}^{-1}(z)\right)}=\frac{1}{\Psi_{\lambda, \eta}\left(\Psi_{\lambda, \eta+1}^{-1}(z)\right)}+\Psi_{\lambda, \eta+1}^{-1}(z)
$$

from where

$$
\Psi_{\lambda, \eta}\left(\Psi_{\lambda, \eta+1}^{-1}(z)\right)=\frac{1}{\frac{1}{z}-\Psi_{\lambda, \eta+1}^{-1}(z)}
$$

Since

$$
\Psi_{\lambda, \eta+1}^{-1}(z)=\frac{1-\lambda z-\sqrt{(1-\lambda z)^{2}-4 z^{2}(\eta+1)}}{2 z(\eta+1)}
$$

by (36)

$$
\Psi_{\lambda, \eta}\left(\Psi_{\lambda, \eta+1}^{-1}(z)\right)=\frac{2 z(\eta+1)}{2 \eta+1+\lambda z+\sqrt{(1-\lambda z)^{2}-4 z^{2}(\eta+1)}} .
$$

Now, (34) follows from (35), (37), and (38).

Remark 2.8. In the free case, it is still an open problem whether one can derive any explicit formulas for the action of $\partial_{\lambda, \eta}^{\dagger}$ on a general function $f$.

Remark 2.9. In [11], a study of a free Meixner class of orthogonal polynomials of infinitely many non-commutative variables has been initiated. We expect that the results of this note related to the free case may be generalized to this infinite dimensional setting, compare with [16]. We hope that this will be discussed in [12]. 
Acknowledgements. We would like to thank Marek Bożejko for numerous useful discussions. EL was partially supported by an LMS Scheme 3 grant and by the PTDC/MAT/67965/2006 grant, University of Madeira.

\section{References}

[1] L. Accardi, U. Franz, and M. Skeide, Renormalized squares of white noise and other non-Gaussian noises as Lévy processes on real Lie algebras, Commun. Math. Phys. 228 (2002) 123-150.

[2] M. Anshelevich, Free martingale polynomials, J. Funct. Anal. 201 (2003) 228-261.

[3] M. Anshelevich, Appell polynomials and their relatives, Int. Math. Res. Not. 2004, no. $65,3469-3531$.

[4] M. Anshelevich, Free Meixner states, Commun. Math. Phys. 276 (2007) 863-899.

[5] M. Anshelevich, Orthogonal polynomials with a resolvent-type generating function, Trans. Amer. Math. Soc. 360 (2008) 4125-4143.

[6] O. E. Barndorff-Nielsen, S. Thorbjørnsen, The Lévy-Itô decomposition in free probability, Probab. Theory Related Fields 131 (2005) 197-228.

[7] Ju. M. Berezanskii, Expansions in Eigenfunctions of Selfadjoint Operators (American Mathematical Society, 1968).

[8] P. Biane, Processes with free increments, Math. Z. 227 (1998) 143-174.

[9] M. Bożejko and W. Bryc, On a class of free Lévy laws related to a regression problem, J. Funct. Anal. 236 (2006) 59-77.

[10] W. Bryc and J. Wesołowski, Conditional moments of $q$-Meixner processes, Probab. Theory Related Fields 131 (2005) 415-441.

[11] M. Bożejko and E. Lytvynov, Meixner class of non-commutative generalized stochastic processes with freely independent values I. A characterization, Arxiv preprint, 2008.

[12] M. Bożejko and E. Lytvynov, Meixner class of non-commutative generalized stochastic processes with freely independent values II. The generating function, in preparation.

[13] T. S. Chihara, An Introduction to Orthogonal Polynomials (Gordon and Breach Sci. Pbl., 1978). 
[14] Y. Kondratiev, J. L. Silva, and L. Streit, Generelized Appell systems, Methods Funct. Anal. Topology 3 (1997), no. 3, 28-61.

[15] Y. Kondratiev, L. Streit, W. Westerkamp, and J. Yan, Generalized functions in infinite dimensional analysis, Hiroshima Math. J. 28 (1998) 213-260.

[16] E. Lytvynov, Polynomials of Meixner's type in infinite dimensions-Jacobi fields and orthogonality measures, J. Funct. Anal. 200 (2003) 118-149.

[17] J. Meixner, Orthogonale Polynomsysteme mit einem besonderen Gestalt der erzeugenden Funktion, J. London Math. Soc. 9 (1934) 6-13.

[18] I. Rodionova, Analysis connected with generating functions of exponential type in one and infinite dimensions, Methods Funct. Anal. Topology 11 (2005) 275-297.

[19] N. Saitoh and H. Yoshida, The infinite divisibility and orthogonal polynomials with a constant recursion formula in free probability theory, Probab. Math. Statist. 21 (2001) 159-170.

[20] W. Schoutens, Stochastic Processes and Orthogonal Polynomials, Lecture Notes in Statist., Vol. 146 (Springer-Verlag, 2000).

[21] P. Śniady, Quadratic bosonic and free white noises, Commun. Math. Phys. 211 (2000) 615-628.

[22] R. Speicher, Free probability theory and non-crossing partitions, Sém. Lothar. Combin. 39 (1997), Art. B39c, 38 pp. (electronic) 